\title{
BMJ Open Relapse in rheumatoid arthritis patients undergoing dose reduction and withdrawal of biologics: are predictable factors more relevant than predictive parameters? An observational prospective real-life study
}

\author{
Olivier Vittecoq (1) , ${ }^{1}$ Sandra Desouches, ${ }^{1}$ Marie Kozyreff, ${ }^{1}$ Julia Nicolau, ${ }^{2}$ \\ Sophie Pouplin, ${ }^{1}$ Pascal Rottenberg, ${ }^{1}$ Nicolas Sens, ${ }^{1}$ Thierry Lequerre, ${ }^{1}$ \\ Gilles Avenel ${ }^{1}$
}

To cite: Vittecoq 0 , Desouches S, Kozyreff M, et al. Relapse in rheumatoid arthritis patients undergoing dose reduction and withdrawal of biologics: are predictable factors more relevant than predictive parameters? An observational prospective real-life study. BMJ Open 2019;9:e031467. doi:10.1136/ bmjopen-2019-031467

- Prepublication history for this paper is available online. To view these files, please visit the journal online (http://dx.doi org/10.1136/bmjopen-2019031467).

Received 08 May 2019 Revised 30 October 2019 Accepted 06 November 2019

Check for updates

(c) Author(s) (or their employer(s)) 2019. Re-use permitted under CC BY-NC. No commercial re-use. See rights and permissions. Published by BMJ.

${ }^{1}$ Rouen University Hospital, Department of Rheumatology, Rouen, France

${ }^{2}$ Rheumatology, Hospital Centre Dieppe, Dieppe, HauteNormandie, France

Correspondence to Dr Olivier Vittecoq; vittecoq.olivier@wanadoo.fr

\section{ABSTRACT}

Objective To determine predictive/predictable factors of relapse in rheumatoid arthritis (RA) patients undergoing biologic Disease-Modifying Anti-Rheumatic Drugs (bDMARDs) dose reduction/discontinuation.

Patients and methods RA patients receiving the same bDMARD for more than 1 year, in Simplified Disease Activity Index (SDAl) remission, were selected in an observational monocentric real-life study. The 18-month follow-up included spacing (6 months) and withdrawal (12 months) periods of bDMARD. Clinical, biological and ultrasonographic (US) parameters were collected regularly. Relapse was defined by SDAl $>11$.

Results Fifty-three RA patients (mean age: 58 years; $72 \%$ women; median duration: 11 years) were enrolled. Forty-two received anti-cytokinic bDMARD targeting tumour necrosis factor $(n=39)$ or interleukin-6R $(n=3)$ and 11 were treated by abatacept. The number of relapses during the spacing and discontinuation periods were 19 and 20, respectively. After 18 months of follow-up, among the 53 patients, 12 maintained bDMARD-free remission, 39 had relapsed and 2 were lost of follow-up. Median time to relapse was 11.8 months. In multivariate analysis, baseline factors predictive of relapse were corticosteroid intake, female gender, longer disease duration and no methotrexate intake with bDMARD. Concerning the survival analysis, also taking into account the factors of predictability, the main risk factor of relapse after discontinuation was an increase of SDAI $>0$ during the spacing period $(p=0.03)$. US findings were not contributive. Conclusion In the context of RA in remission under bDMARDs, variation of SDAl during the dose-reduction phase is more relevant than baseline parameters to predict success of drug withdrawal.

\section{INTRODUCTION}

In rheumatoid arthritis (RA), after achieving low disease activity (LDA) or remission, ${ }^{1}$ the goal of therapy is to maintain clinical,
Strengths and limitations of this study

- This prospective real-life multiparameter study used a well-defined procedure of gradual dose reduction and discontinuation of biologic Disease-Modifying Anti-Rheumatic Drugs (bDMARDs) in rheumatoid arthritis patients in remission for at least 1 year.

- Very strict remission criteria based on clinical and ultrasonographic data were required to consider dose reduction of bDMARDs.

Both factors of prediction (collected just prior to therapeutic relief) and predictability (related to kinetic of parameters during the dose-reduction phase) were considered as potential predictors of relapse.

- Almost all bDMARDs were taken into account to identify candidate predictors of relapse after discontinuation that could be applied to any drug.

- The main weakness of this study is the limited population size.

functional and structural remission. ${ }^{2}$ For some patients, this is possible even after the cessation of biologic Disease-Modifying Anti-Rheumatic Drugs (bDMARD). ${ }^{3}$ The opportunity of discontinuing bDMARD after achieving remission must be considered because of potential long-term safety issues and the economic burden associated with their expense. Furthermore, the disease can spontaneously evolve towards an inactive form. Multiple studies have investigated whether remission can be sustained after a bDMARD is discontinued, namely, biologicfree remission (BFR). ${ }^{3}$

The European League Against Rheumatism (EULAR) 2012 guidelines ${ }^{4}$ suggests that we can consider tapering bDMARD. 
Determining the patient profile associated with a high chance of sustained remission after the cessation of bDMARD is of great importance to avoid disease flares. For this purpose, two definitions of remission have been proposed, either the Boolean definition or a score of the Simplified Disease Activity Index $(\mathrm{SDAI})<3.3 .{ }^{56}$ However, the majority of studies did not use these definitions for eligibility to bDMARD spacing or withdrawal. Indeed, in most reports, Disease Activity Score on 28 joints (DAS 28) was used to select patients for withdrawal of bDMARD in RA patients having achieved remission. ${ }^{7}$

For patients with long-standing RA, the discontinuation of tumour necrosis factor (TNF) inhibitors after sustained remission has been shown to be possible in some cases. However, high flare rates have been documented in other studies. For these patients, bDMARD dose reduction or spacing regimen followed by secondary withdrawal may be preferable instead of sudden discontinuation. ${ }^{3}$

According to several studies, it appears that the criteria for spacing the administration of bDMARD in RA patients in remission are not consensual and that we lack validated data. In this respect, a systematic review of studies addressing predictors of successful dose reduction or discontinuation of bDMARD in RA shows that there is no consistent predictor. ${ }^{7}$

To respect EULAR recommendations, we introduced standardised practices in our rheumatology department, in routine care, several years ago. Spacing and then discontinuation of bDMARD is performed in RA patients in remission according to 2011 ACR/EULAR criteria. ${ }^{6}$

The general objective of this real-life, prospective study was to define strict eligibility criteria for bDMARD spacing/withdrawal in long-standing RA patients in remission. The specific objectives were (1) to define the rate of relapse during the spacing and withdrawal periods in a RA population; (2) to identify predictive/predictable factors of relapse during the withdrawal phase of bDMARD and (3) to determine whether duration and degree of clinical remission as well as ultrasonographic (US) findings at time of bDMARD spacing influenced the achievement of bDMARD withdrawal.

\section{PATIENTS AND METHODS}

\section{Study design}

This prospective real-life study comprised an inclusion visit and two phases (figure 1).

\section{Patients}

In this study all RA patients, who were enrolled, were treated by bDMARD between 2012 and 2014, in the rheumatology department of Rouen University Hospital. bDMARDs were infliximab, etanercept, adalimumab, abatacept, certolizumab and tocilizumab. Golimumab was not considered since it was introduced more recently. Rituximab was not relevant for such a strategy of spacing/ withdrawal. Patients with subcutaneous treatment were selected at annual follow-up visits in the ambulatory care unit. Patients with intravenous bDMARD were selected in the immunotherapy unit of the department.

\section{Inclusion criteria}

They comprised RA patients (older than 18 years), fulfilling ACR/EULAR 2010 criteria, in remission defined as a DAS $28<2.6$ for at least 12 months, and receiving the same bDMARD for at least 1 year. Prior to initiation of spacing, a SDAI $<3.3$ was required. ${ }^{6}$ Patients taking prednisone (or equivalent) at a dose $>5 \mathrm{mg}$ /day or with structural evolution during the previous year were excluded.

\section{Ethics}

The agreement of both hospital and private rheumatologists was collected before bDMARD spacing. All patients gave their consent for this procedure.

\section{Schedule of visits and dose tapering}

All visits were planned every 2 months during the spacing phase that lasted 6 or 7 months according to the bDMARD used and then every 3 months during 1 year after discontinuation of the bDMARD. Dose tapering was standardised for each drug during the spacing phase as shown in figure 1 .

\section{Parameters studied}

\section{Inclusion visit}

During this visit, the presence of all inclusion criteria was checked. The following parameters were collected: all data needed to calculate DAS 28-ESR, DAS 28-CRP and SDAI; completion of the Health Assessment Questionnaire (HAQ); laboratory tests (erythrocyte sedimentation rate (ESR), C-reactive protein (CRP), rheumatoid factors (RF) and anti-cyclic citrullinated peptide (anti-CCP) antibodies). We also recorded the following data for each patient: demographic characteristics, RA duration, number of synthetic DMARDs, bDMARDs received and time on bDMARD.

In addition, US examination of the DAS 28 joints (US-DAS 28) was carried out at baseline, using a MyLab 70 (Technos Esaote), by four operators (MK, JN, PR, NS) with long-standing experience in US evaluation of chronic inflammatory rheumatic diseases. They have already participated in several multicenter studies and the intra-observer and inter-observer reliability was similar to that reported in the study conducted by D'Agostino $e t a l^{8}$ All sonographers were blinded to clinical information and laboratory data. A systematic multiplanar gray-scale (GS) and power-Doppler (PD) US-DAS 28 was performed using a high-frequency $(13.5 \mathrm{MHz})$ linear array transducer. Joints were evaluated using a semi-quantitative scoring system with a 0-3 scale for GS and PD according to the method developed by Szkudlarek et al. ${ }^{9}$ Findings were described using the definitions established by the OMERACT. The overall GS and PD scores for synovitis were measured and the global PDUS score (sum of total GS and PD scores) was calculated for each patient. 


\section{Consecutive RA patients in clinical remission (DAS28 ESR $<2.6$ ) on} biologic agent for at least 1 year during the 2012-2014 study period

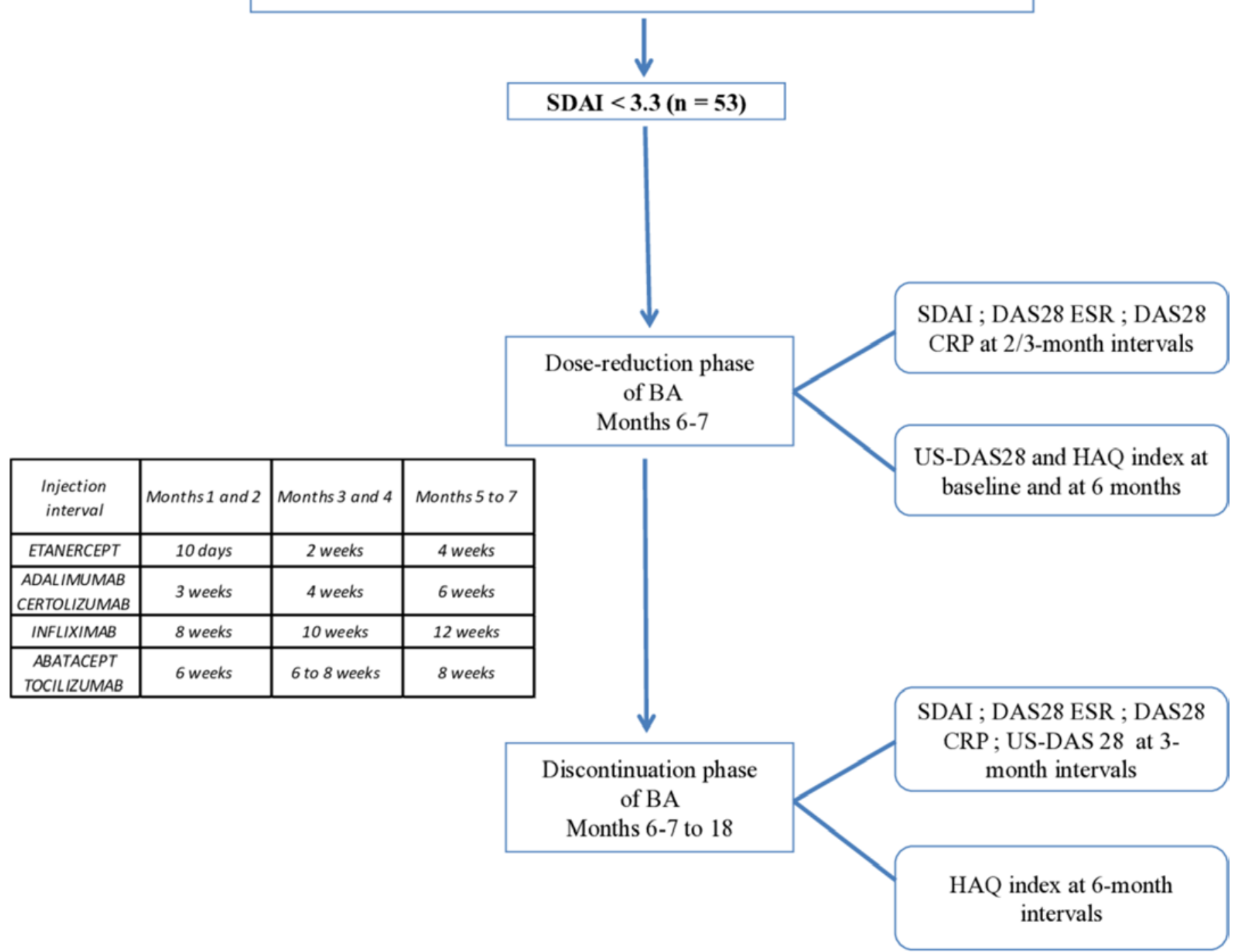

Figure 1 Study design. CRP, C-reactive protein; DAS, Disease Activity Score; ESR, erythrocyte sedimentation rate; HAQ, Health Assessment Questionnaire; RA, rheumatoid arthritis; SDAI, Simplified Disease Activity Index; US-DAS, ultrasound Disease Activity Score.

\section{Spacing procedure}

A visit was scheduled every 2 or 3 months according to the bDMARD. At each visit, the parameters were mentioned previously and ongoing treatments were recorded. An US-DAS 28 was carried out at month 7 .

Spacing was defined for each bDMARD. During this period, visits were performed at three time points: months 2, 4 and 6 or 7. The inter-injection interval was increased at each visit in order to stop bDMARD completely at month 7 .

During the study period, all associated treatments were unmodified. The dose of conventional DMARDs and corticosteroids was stable.

\section{Follow-up visits after bDMARD discontinuation}

After discontinuation at month 7, patients were evaluated at 3 month intervals via physical examination, ESR and
CRP determinations, SDAI and DAS 28 computation and 28 joint US examination.

\section{Definition of relapse}

Relapse was defined as SDAI $>11$ which was determined by rheumatologists who were blinded to US findings.

In this dose-reduction phase, patients restarted their treatment with the previous scheme. After discontinuation, relapsing patients were immediately retreated with their previous bDMARD, at the previous dosage, with no change in prednisone or synthetic DMARD dosage.

\section{Statistical analysis}

Demographic characteristics, clinical and biological data were summarised by descriptive analysis. Student's t-tests and Fisher's tests were used for quantitative and qualitative variables, respectively. Relapse-free survival data were 
analysed using the log-rank test. Qualitative variables were analysed directly; quantitative variables have been expressed as compared with normal values or median; $p$ values lower than 0.10 were considered significant to be analysed in a multivariate model. In multivariate survival analysis, the Cox model was used. We used NCSS V.2007 for statistical analysis. $\mathrm{P}$ values lower than 0.05 were considered significant.

Candidate predictors were age, gender, disease duration, immunological status, number of previous bDMARDs, type of bDMARD, treatments combined with bDMARD and their dose, DAS at baseline and their kinetic during the spacing phase, US data at baseline and their outcome during the dose-reduction phase, HAQ at baseline and its kinetic during the tapering phase, ESR, CRP.
Two types of analysis were performed. For the first one, the primary outcome was relapse versus nonrelapse either during the dose-reduction phase or over the discontinuation period. The second one, which was the more relevant, was focused on time to relapse. Concerning the two patients in remission at their last visit who were lost of follow-up, they have been censored at the time of their last visit. Thus, they have been included in the remission group for the survival analysis but excluded from the binary outcome analysis of relapse versus non-relapse.

\section{Patient and public involvement}

Patients were not involved in the design and the conduction of the present study.

Table 1 Demographic, clinical, biological and drug characteristics of the study population at baseline and according to the occurrence of relapse during the spacing and discontinuation phases

\begin{tabular}{|c|c|c|c|c|c|c|c|}
\hline \multirow[b]{2}{*}{ Parameter } & \multirow[b]{2}{*}{$\begin{array}{l}\text { Total } \\
(n=53)\end{array}$} & \multicolumn{3}{|c|}{ Spacing phase } & \multicolumn{3}{|c|}{ Discontinuation phase } \\
\hline & & $\begin{array}{l}\text { Non-relapser } \\
(n=34)\end{array}$ & $\begin{array}{l}\text { Relapser } \\
(n=19)\end{array}$ & $P$ value & $\begin{array}{l}\text { Non-relapser } \\
(n=13)\end{array}$ & $\begin{array}{l}\text { Relapsers } \\
(\mathrm{n}=38)\end{array}$ & $P$ value \\
\hline Gender, female n (\%) & $38(72)$ & $20(59)$ & $18(95)$ & 0.009 & $7(54)$ & $30(79)$ & 0.14 \\
\hline Age, median (IQR) & $58(49-63)$ & $58(50-63)$ & $56(48,5-61)$ & 0.56 & $52(47-62)$ & $58(50-63)$ & 0.52 \\
\hline Disease duration, median (IQR) & $11(6-15)$ & $10(6.3-12.8)$ & $12(7-16)$ & 0.23 & $9(8-11)$ & $12(6.3-15)$ & 0.29 \\
\hline bDMARD duration, median (IQR) & $5(3-8)$ & $4(3-7)$ & $7(3.5-8)$ & 0.07 & $4(3-7)$ & $5.5(3-8)$ & 0.38 \\
\hline DAS 28 ESR, median (IQR) & $1.8(1.4-2.1)$ & $1.7(1.4-2.1)$ & $2(1.4-2.2)$ & 0.62 & $1.5(1.3-1.9)$ & $2(1.5-2.2)$ & 0.11 \\
\hline DAS 28 CRP, median (IQR) & $1.6(1.2-1.9)$ & $1.6(1.2-1.9)$ & $1.6(1.4-1.8)$ & 0.93 & $1.7(1.5-1.9)$ & $1.6(1.2-1.8)$ & 0.18 \\
\hline SDAI, median (IQR) & $2(1-3)$ & $1.6(0.8-3)$ & $2(1-3.1)$ & 0.28 & $2.4(2-3)$ & $1(1-2.9)$ & 0.13 \\
\hline HAQ (0-3), median (IQR) & $0.1(0-0.3)$ & $0.1(0-0.3)$ & $0.1(0-0.3)$ & 0.85 & $0.1(0-0.3)$ & $0.1(0-0.3)$ & 0.84 \\
\hline RF positivity (>20IU/mL), n (\%) & $33(62)$ & $19(56)$ & $14(74)$ & 0.25 & $8(62)$ & $24(63)$ & 1 \\
\hline $\begin{array}{l}\text { Anti-CCP positivity (>10UA } / \mathrm{mL}) \text {, } \\
n(\%)\end{array}$ & $33(62)$ & $24(71)$ & $9(47)$ & 0.14 & $9(69)$ & $22(58)$ & 0.52 \\
\hline GS score, median (IQR) & $0(0-2)$ & $0(0-2)$ & $0(0-0)$ & 0.39 & $0(0-0)$ & $0(0-2)$ & 0.37 \\
\hline PD score, median (IQR) & $0(0-0)$ & $0(0-0)$ & $0(0-0)$ & 0.59 & $0(0-0)$ & $0(0-0)$ & 0.5 \\
\hline \multicolumn{8}{|l|}{ bDMARD } \\
\hline Certolizumab, n (\%) & $1(2)$ & $1(3)$ & $0(0)$ & 0.87 & $0(0)$ & $0(0)$ & 0.22 \\
\hline Etanercept, n (\%) & $24(45)$ & $13(38)$ & $11(58)$ & & $5(38)$ & $18(47)$ & \\
\hline Adalimumab, n (\%) & $8(15)$ & $6(18)$ & $2(11)$ & & $1(8)$ & $7(18)$ & \\
\hline Abatacept, n (\%) & $11(21)$ & $8(24)$ & $3(16)$ & & $2(15)$ & $9(24)$ & \\
\hline Infliximab, n (\%) & $6(11)$ & $4(12)$ & $2(11)$ & & $3(23)$ & $3(8)$ & \\
\hline Tocilizumab, n (\%) & $3(6)$ & $2(6)$ & $1(5)$ & & $2(15)$ & $1(3)$ & \\
\hline \multicolumn{8}{|l|}{ Methotrexate } \\
\hline $\mathrm{N}(\%)$ & $42(79)$ & $28(82)$ & $14(74)$ & & $12(92)$ & $28(74)$ & 0.25 \\
\hline Dose, median (IQR) & $15(7.5-15)$ & $15(7.5-16.9)$ & $10(8.8-15)$ & 0.47 & $15(10-25)$ & $10(5.6-15)$ & 0.24 \\
\hline \multicolumn{8}{|l|}{ Prednisone } \\
\hline $\mathrm{N}(\%)$ & $4(8)$ & $2(6)$ & $2(11)$ & & $0(0)$ & $4(11)$ & 0.56 \\
\hline Dose, median, IQR & $0(0-0)$ & $0(0-0)$ & $0(0-0)$ & 0.53 & $0(0-0)$ & $0(0-0)$ & 0.23 \\
\hline
\end{tabular}

anti-CCP, anti-cyclic citrullinated peptide; bDMARD, biologic Disease-Modifying Anti-Rheumatic Drugs; CRP, C-reactive protein; DAS, Disease Activity Score; ESR, erythrocyte sedimentation rate; GS, grey scale; HAQ, Health Assessment Questionnaire; IQR, Interquartile range; PD, power Doppler; RF, rheumatoid factors; SDAI, Simplified Disease Activity Index. 


\section{RESULTS}

\section{Baseline characteristics of the study population}

Among the 378 RA patients treated with a bDMARD between January 2012 and January 2013, 53 (14\%) fulfilled our criteria for disease remission (SDAI <3.3) and were selected for the spacing/discontinuation standardised procedure (figure 1). This cohort included 38 female and 15 male patients with a mean age of 58.5 years, a mean disease duration of 13 years (median 11 years; $4-32$ years); $62 \%$ patients were RF positive and $62 \%$ were anti-CCP positive; $49 \%$ were double positive and $25 \%$ double negative; $85 \%$ patients had at least one X-ray erosion. Among double-negative RA patients, $85 \%$ had structural damage. At the inclusion visit, the mean values of DAS 28-ESR, DAS 28-CRP, SDAI and HAQ were 1.76, $1.6,1.9$ and 0.23 , respectively.

Among the 53 patients, $6,8,24,11,1$ and 3 were on infliximab, adalimumab, etanercept, abatacept, certolizumab and tocilizumab, respectively; 10 patients were switched to another bDMARD: 1 switch $(\mathrm{n}=5), 2$ switches $(n=3), 3$ switches $(n=2)$. At the time of the study, 42 $(79.2 \%)$ patients were taking methotrexate at a mean dose of $11.79 \mathrm{mg} /$ week, and 1 patient was on leflunomide. Thus, 10 patients received bDMARD in monotherapy. Only four patients were on prednisone (mean dose: $3.13 \mathrm{mg} /$ day).

Results are also expressed in median (IQR) and summarised in table 1.

US data are shown in table 2. The mean score of GS synovitis was 1.7 and that of PD synovitis was 0.7 . The mean global score was 2.5, reflecting LDA. Among the 53 patients, $38(72 \%)$ had a global score of 0 . Global scores for GS and PD assessments were also expressed in a Boolean manner according to the definition used for the four items of the last remission criteria $(<\mathrm{or}=1 / 28) .{ }^{6}$ Each joint was graded 0 or 1 . A value of 1 was considered when the grade was $>1$ (2 or 3 ) for a given joint according to the Szkudlarek definition. ${ }^{7}$ Using this Boolean definition for US-DAS 28, 3 quarters of patients had a GS score $\leq 1$ and, more importantly, $89 \%$ had a PD score $\leq 1$ at baseline (table 2 ).

\section{Spacing and discontinuation periods}

During the spacing period, 5 patients relapsed at month 4 and 14 at month 7 .

Thirty-four patients were able to stop their bDMARD at month 7. At month 9, 7 patients relapsed, and 27 were able to continue treatment withdrawal. Ten patients relapsed at month 12, 2 at month 15 and 2 at month 18 . At the end of the 18 months follow-up period, 14 patients had completed the visit; 2 had relapsed while 12 were still in remission. Among the 53 patients, 41 relapsed. Among those who relapsed, there were two patients in remission at their last visit who were lost to follow-up. Importantly, all patients on monotherapy (without combination with methotrexate) relapsed, as well as the four patients who received a low dose of corticosteroids.

Among the 12 non-relapsing patients, the mean DAS 28 CRP was 2.14 (1.43-2.86; SD: 0.7) and the mean SDAI was $4.03(0.37-7.7$, SD: 3.66$)$ at the last visit. Ten had a DAS 28 CRP $<2.6$ and 7 had a SDAI $<3.3$ at all visits. Thus, according to the SDAI definition of remission, only seven patients had a sustained deep remission (ie, a SDAI $<3.3$ at all time points).

During the spacing and discontinuation phases, there were more patients with a global PD score $>1 / 28$ according to the Boolean definition (table 2).

\section{Identification of predictive factors of relapse}

The survival analysis of patients who relapsed found a median relapse time of 11.8 months (figure 2). There were a majority of women in the relapsing group: $79.5 \%$ vs $50 \%$ in non-relapsers $(p=0.066)$. The proportion of patients with disease duration longer than the median (11 years) was significantly higher in the relapsing group: $56.4 \%$ of relapsers versus $16.7 \%$ of non-relapsers $(p=0.022)$. Age, anti-CCP or RF positivity/titers were not significantly different between relapsers and non-relapsers. Indeed, the number of non-relapsers who were seronegative and without erosions was limited to one patient. Clinical and US composite scores showed no significant difference. In this regard, while all patients with at least one PD-positive-US synovitis (grade $>1$ ) were relapsers, those with a global score of 0 on sonography or satisfying the Boolean

\begin{tabular}{|c|c|c|c|c|c|c|c|c|c|c|c|c|}
\hline \multirow{2}{*}{$\begin{array}{l}\text { Sonography } \\
\text { Number of } \\
\text { patients }\end{array}$} & \multicolumn{2}{|l|}{ Baseline } & \multicolumn{2}{|c|}{ Month 6-7 } & \multicolumn{2}{|l|}{ Month 9} & \multicolumn{2}{|c|}{ Month 12} & \multicolumn{2}{|c|}{ Month 15} & \multicolumn{2}{|c|}{ Month 18} \\
\hline & 53 & & 42 & & 30 & & 20 & & 15 & & 12 & \\
\hline Mode & GS & PD & GS & PD & GS & PD & GS & PD & GS & PD & GS & PD \\
\hline Score $=0$ & $20(38)$ & $36(68)$ & $15(36)$ & $26(62)$ & $6(20)$ & $14(47)$ & $7(25)$ & $13(65)$ & $3(20)$ & $8(53)$ & $2(17)$ & $7(58)$ \\
\hline Score $=1$ & $19(36)$ & $11(21)$ & $11(26)$ & 7 (17) & $8(27)$ & $6(20)$ & $0(0)$ & $2(10)$ & $5(33)$ & $0(0)$ & $5(42)$ & $2(17)$ \\
\hline Score $\leq 1$ & $39(74)$ & 47 (89) & $26(62)$ & $33(79)$ & $14(47)$ & $20(67)$ & $7(25)$ & $15(75)$ & 8 (53) & $8(53)$ & 7 (59) & $9(75)$ \\
\hline Score $>1$ & $14(26)$ & $6(11)$ & $16(38)$ & $9(21)$ & $16(53)$ & $10(33)$ & $13(75)$ & $5(25)$ & $7(47)$ & $7(47)$ & $5(41)$ & $3(25)$ \\
\hline
\end{tabular}

Results are expressed as number (\%).

GS, grey scale; PD, power Doppler. 


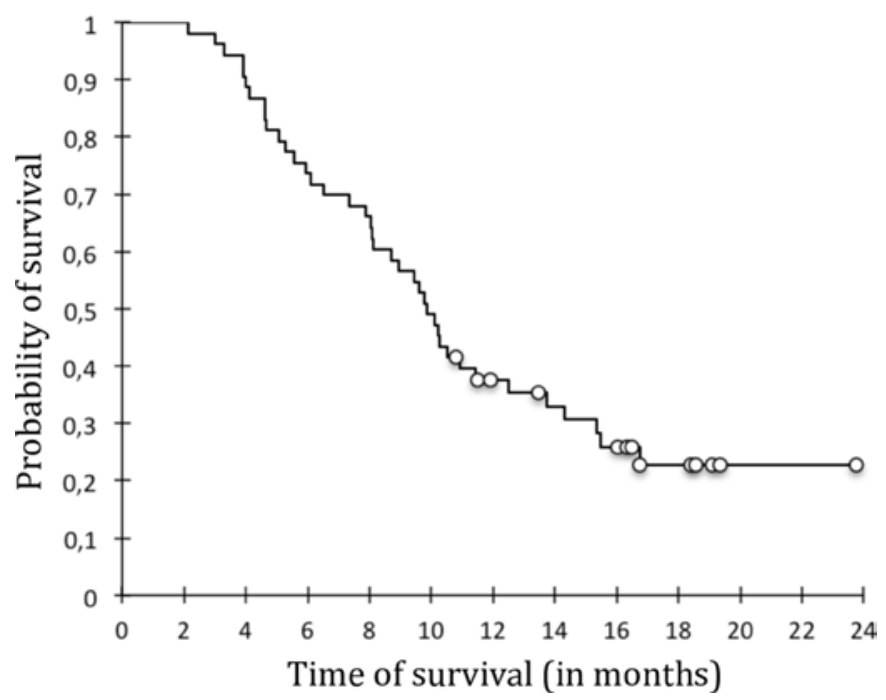

Figure 2 Global survival curve of biologic free remission.

definition for GS or PD global scores could be relapsers or non-relapsers.

Survival analysis between relapsers and non-relapsers showed that the following criteria disease duration longer than the median $(\mathrm{p}=0.032)$, previous biologic therapy $(p=0.068)$, treatment with corticosteroids $\left(p=10^{-3}\right)$, ESR $>10(p=0.098)$ were significantly (or tended to be) associated with relapse.

In multivariate analysis, relapse risk factors were corticosteroid use with a risk ratio of relapse at 13.78 (95\% CI 3.95 to $48.08, p=0.001$ ), disease duration longer than the median (11 years) with a risk ratio at 2.18 (95\% CI: 1.08 to $4.39, \mathrm{p}=0.029)$.

\section{Risk factors of relapse taking into account both factors of prediction and of predictability}

This analysis comprises both baseline parameters collected prior to dose-reduction phase (referred to as predictive factors) and kinetic of clinical, biological and US parameters during the tapering phase prior to discontinuation (labelled factors of predictability).

It has been carried out from patients who completed the tapering phase and underwent the discontinuation period.

Survival analysis during the withdrawal period, taking into account baseline parameters and the evolution of some of them during the spacing phase, was performed and included 32 patients. Median survival was 7.6 months (5.3-11.2) following the month 7 visit. There were 12 non-relapsing patients whereas 20 patients relapsed.

The univariate analysis showed that disease duration longer than the median $(p=0.021)$ was predictive of relapse after discontinuation of treatment. Before spacing, methotrexate intake $(\mathrm{p}=0.140)$ was a potential protective factor during discontinuation of treatment.

The univariate survival analysis, taking into account the kinetics of parameters between month 0 (baseline) and month 7 (end of spacing phase), showed that variations

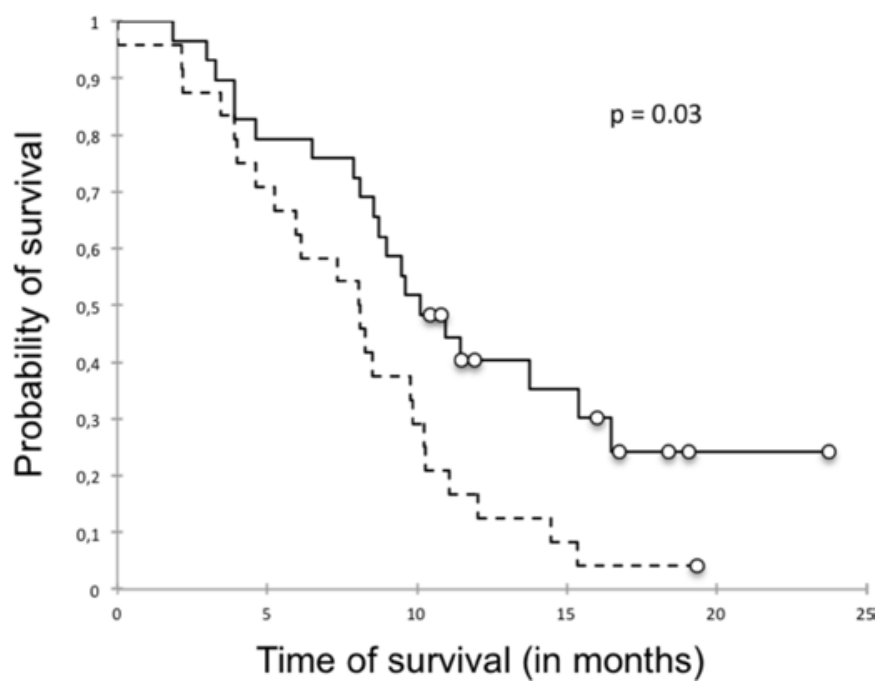

Figure 3 Survival curve showing that a SDAI increase $>0$ during the spacing phase was significantly associated with a higher risk of relapse. Survival curve of delta SDAl $>0$ (dotted line) and delta SDAI $\leq 0$ (full line). SDAI, Simplified Disease Activity Index.

of SDAI was significantly associated with relapse (figure 3) in contrast to those of US scores (table 3).

The multivariate analysis identified as relapse risk factors a SDAI increase $>0$ between month 0 and month 7 with a risk ratio of 21.77 (95\% CI 2.1 to $225.74, p=0.03)$ (figure 3). This point means that an increase of SDAI between two visits during the tapering phase was predictive of relapse defined by a SDAI $>11$ after bDMARD discontinuation. In contrast, methotrexate use was protective of relapse with a risk ratio of 0.07 (95\% CI 0.01 to $0.61, \mathrm{p}=0.016)$. After exclusion of the four patients who received low doses $(1,2.5,4$ and $5 \mathrm{mg}$ /day) of corticosteroids, the same findings were obtained (data not shown).

\section{DISCUSSION}

Our study has several strengths. This was a prospective reallife study with a standardised procedure for spacing and discontinuation in accordance with international recommendations. ${ }^{4}$ Our analysis took into account all bDMARD available except rituximab and JAK/STAT inhibitors (not available at the time of analysis), unlike other analyses that focused mainly on TNF-blocking agents or a single bDMARD (infliximab, etanercept, adalimumab, tocilizumab, abatacept). Even though the sample size for patients treated with abatacept and tocilizumab was low, we did not perform any specific analysis focused on TNF blockers since we consider that candidate predictors of relapse after discontinuation should be applied to any bDMARD, whatever its mechanism of action. Moreover, although they target TNF, all TNF antagonists have their own specificities concerning the mode of action.

Our study is one of the first to consider factors of predictability. However, one limitation of our study is the limited population size. These 53 patients in remission represented $14 \%$ of our population of 378 RA patients. 
Table 3 Analysis of kinetic of composite indexes and ultrasonographic data during the spacing phase as potential predictable factors of relapse after biologic agent withdrawal

\begin{tabular}{lllll}
\hline Population & All & Relapser & Non-relapser & P value \\
\hline Composite indexes & & & & \\
Delta DAS 28 ESR > 0, \% & 77 & 82.4 & 66.7 & 0.18 \\
\hline Delta DAS 28 ESR, mean (SD) & $0.4(0.56)$ & $0.56(0.57)$ & $0.19(0.49)$ & 0.07 \\
\hline Delta DAS 28 CRP > 0, \% & 48 & 52.9 & 37.5 & 0.149 \\
\hline Delta DAS 28 CRP, mean (SD) & $0.1(0.62)$ & $0.22(0.66)$ & $-0.07(0.5)$ & 0.582 \\
\hline Delta SDAI > 0, \% & 60 & 70.6 & 37.5 & 0.03 \\
Delta SDAl, mean (SD) & $0.8(2.87)$ & $1.39(3.17)$ & $-0.34(1.69)$ & 0.03 \\
Ultrasonographic data & & & & \\
Delta GS score > 0, \% & 17.4 & 12.5 & 28.6 & 0.55 \\
\hline Delta GS score, mean (SD) & $0(0.98)$ & $-0.19(0.98)$ & $0.29(0.95)$ & 0.39 \\
\hline Delta PD score > 0, \% & 30.4 & 31.3 & 28.6 & 1 \\
Delta PD score, mean (SD) & $0.1(1.01)$ & $0.06(1.06)$ & $0.29(0.95)$ & 0.68 \\
\hline Delta global score > 0, \% & 21.7 & 18.8 & 28.6 & 0.62 \\
Delta global score, mean (SD) & $-0.4(5.01)$ & $-1.06(5.8)$ & $1.14(1.95)$ & 0.16 \\
\hline
\end{tabular}

CRP, C-reactive protein; DAS, Disease Activity Score; ESR, erythrocyte sedimentation rate; GS, grey scale; PD, power Doppler; SD, standard deviation; SDAI, Simplified Disease Activity Index.

This small percentage of patients in remission may be related to the strict definition of remission that we used (SDAI <3.3) whereas, when DAS 28-ESR was considered (DAS 28<2.6), $142(38 \%)$ patients were in remission, which is more in line with data reported in the literature. In addition, the majority of RA patients in our unit had long-standing disease. Moreover, the tapering strategy was very rapid compared with those reported in the literature or done in daily practice but, to our knowledge, there is no consensus about the dose-reduction process for each bDMARD. Nevertheless, this might alter the external validity of the present results. Finally, we have not investigated molecular and cellular biomarkers likely to reflect an immunological remission.

The characteristics of our population are concordant with those observed in other reports such as PRESERVE, BEST, PRIZE, STRASS and that of Brocq et al. ${ }^{10-14}$

Based on EULAR recommendations ${ }^{4}$ and data from the literature, we performed spacing of bDMARD rather than sudden discontinuation. Our scheme has the distinction of proposing gradual spacing and then discontinuation as in the STRASS study in which bDMARD were represented by etanercept and adalimumab. ${ }^{14}$ Other studies (BeSt, PRESERVE, PRIZE) proposed dose reduction. ${ }^{9} 1013$ In the present study and in the STRASS study, ${ }^{14} 35.8 \%$ and $26.5 \%$ of patients relapsed, respectively, during the spacing period; then $64 \%$ and $37.5 \%$ of patients were able to stop bDMARD and finally $77 \%$ and $81 \%$ relapsed, respectively.

Our study required very strict remission criteria compared with other studies and notably had a remission duration of at least 1 year compared with other studies which often selected patients with a remission duration of 6 months. In addition, we defined remission by SDAI $<3.3^{6}$ when other studies (RRR, PRESERVE) used DAS 28 $<3.2^{1015}$ or DAS $28<2.6 .{ }^{12-1416}$ In this respect, with LDA as criteria of selection (DAS $28<3.2$ ), RRR and PRESERVE had a lower bDMARD-free remission rate ( $43 \%$ and $42 \%$ ) than PRIZE and BeSt (53\% and 80\%). Thus, it seems better to use bDMARD withdrawal only in patients with deep remission as reported by Tanaka $e t$ al who found that a DAS 28 ESR $<2.2$ was associated with maintenance of DFR. ${ }^{15}$ Those data led us to retain a SDAI $<3.3$ as a criterion of eligibility for bDMARD spacing. Such a level of clinical and biological remission is close to US remission as observed in a previous study ${ }^{17}$ and in ours in which three quarters of patients had a global (GS plus PD) US-DAS 28 score of 0 . Moreover, the duration of remission appears to be an important prerequisite to consider bDMARD relief. Indeed, subclinical joint activity is longlasting in RA joints in clinical remission. Even though there is attenuation over time, the mean time $( \pm \mathrm{SD})$ since last clinical swelling and positive sonographic assessment was significantly shorter in patients showing high GS or PD signals compared with lower-grade GS or PD signals. ${ }^{18}$ Since subclinical disease activity may persist several years in clinically inactive joints and US PD-positive synovitis is related to subsequent flare, ${ }^{19-21}$ deep remission based on US-DAS 28 findings is also required.

Nevertheless, deep remission based on the absence of PD-positive synovitis (89\% in the present study) and on US-DAS 28 seems insufficient to predict BFR since a large proportion of patients with a global US score of 0 were relapsers. We can postulate that a single evaluation prior to bDMARD relief is not relevant enough and thus sequential assessment at regular intervals during a period 
that needs to be defined should be performed to confirm that US remission is persistent before initiating bDMARD dose reduction. In this respect, in the study conducted by Alivernini $e t a l$, the selection of patients was based on US findings in a cohort of 42 consecutive patients with long-standing RA in clinical remission (DAS $<1.6$ for at least 6 months) and receiving combination therapy with methotrexate and TNF-blocking agents (adalimumab or etanercept). Despite serial PD-negative findings during the tapering and discontinuation phases, $38 \%$ of patients relapsed after 12 months follow-up after discontinuation. ${ }^{22}$

Since characteristics of remission prior to bDMARD relief are unable to predict BFR, the question arose as to whether other parameters, before bDMARD discontinuation as well as during the spacing phase, were potential risk factors of relapse after bDMARD withdrawal.

One of the predictive factors of relapse was longstanding RA with disease duration longer than the median (11 years). In fact, most studies ${ }^{10111315}$ focused on more recent RA with a disease duration of less than 6 years. The populations closest to ours were those of the Brocq and STRASS ${ }^{12} 14$ in which the mean disease duration was 11 and 9 years, respectively. These two studies analysed non-naive bDMARD patients and the remission rate at 1 year was $24 \%$ and $37.5 \%$, respectively, which was closer to that of our cohort (23\%) but lower than that observed in studies with shorter disease duration that included naive bDMARD RA (PRESERVE: 42\%, BeSt: $80 \%$, PRIZE: 50\%, RRR: 43\%). ${ }^{1011} 1315$ Thus, our results are in line with data in the literature since a disease duration longer the threshold of 5 years is a factor of relapse. In the same way, use of previous bDMARD is a risk factor of relapse.

Low-dose glucocorticoid, less than $5 \mathrm{mg}$ /day, was still associated with relapse after treatment discontinuation. Spacing or discontinuation could not be initiated in patients with glucocorticoids, even at a very low dose ( $3 \mathrm{mg} /$ day in the present study). RRR and STRASS studies allowed corticosteroids at a dose less than $5 \mathrm{mg} /$ day, ${ }^{1415}$ PRESERVE tolerated up to $10 \mathrm{mg}$ /day with $60 \%$ of patients on prednisone. ${ }^{10}$ These studies did not observe a correlation between relapse and long-term corticosteroid. For EULAR, in patients in long-term remission the first step is to reduce corticosteroids, and in case of persistent remission the next step is to decrease bDMARD.

Pertinently, the combination with a synthetic DMARD is of importance for the success of bDMARD discontinuation. Indeed, methotrexate combination with bDMARD is a protective factor of relapse. While Brocq et al did not find it, BeSt reported a protective effect of methotrexate. ${ }^{1112}$ The same findings were stated in the PRESERVE and PRIZE studies. ${ }^{10} 13$ In a meta-analysis, ${ }^{23}$ a combination of methotrexate with bDMARD achieved LDA more quickly and ensured the maintenance of remission after discontinuation of bDMARD, more likely in case of monotherapy. In a meta-analysis of randomised controlled trials on stopping bDMARDs in monotherapy, relapse was observed in $46 \%$ of RA patients after discontinuation of bDMARDs. ${ }^{3}$ There are no consensual guidelines for bDMARD discontinuation. NICE recommends a prudent decrease in the dose of bDMARDs with a recovery to previous dose in case of relapse. ${ }^{24}$ EULAR recommends that bDMARD tapering can be considered if a patient is in persistent remission after glucocorticoid tapering, especially if this treatment is combined with conventional DMARDs such as methotrexate. ${ }^{4}$ For EULAR, spacing treatment or decreasing the dosage is quite similar. Those guidelines corroborate our results.

Although data from the RETRO, BeSt, HIT-HARD and POET studies suggest that anti-CCP status has an influence on relapse with a lower chance of maintaining remission in the presence of anti-CCP, ${ }^{25}$ positivity and/ or levels of markers reflecting systemic inflammation or autoimmunity (RF and anti-CCP) were not predictive of relapse in the present study. Only composite biomarker testing including acute phase reactants, cytokines and metalloproteinases were suggested to be relevant in the RETRO study. ${ }^{26}$

While there was no difference between a DAS 28 $<2.6$ and a SDAI $\leq 3.3$ on relapse after stopping treatment, a fluctuation of $>0$ in SDAI during spacing was significantly associated with relapse. There are no data in the literature on the influence of DAS 28 or SDAI fluctuations on relapse during the spacing period. However, although SDAI at baseline showed no difference, we consider this score to be more robust than DAS 28 and our analysis shows that a worsening of this score was associated with relapse. This analysis provides new criteria for tapering bDMARD. Given our results, only patients with decreased SDAI score in relief can stop bDMARD, whereas other patients with increased SDAI should continue bDMARD at the last dose or injection interval. Thus, the kinetic of SDAI during the spacing period seems to be more important than baseline values.

\section{CONCLUSION}

We propose spacing of bDMARD for patients with RA of limited duration, in both clinical and US deep remission of at least 1 year, on conventional DMARDs, especially methotrexate and after tapering corticosteroids. Therefore, we suggest withdrawal of bDMARD only if the SDAI or DAS does not worsen during the spacing period.

Other studies are needed to confirm the relevance of these predictive and predictable factors of relapse when considering bDMARD alleviation/discontinuation in RA patients in remission under bDMARD and to evaluate the interest of a panel of molecular and cellular biomarkers that could help to personalise DMARD withdrawal as suggested by recent works using composite scores or multi-omics approaches to define molecular remission. ${ }^{27} 28$

Acknowledgements We are grateful to Nikki Sabourin-Gibbs, Rouen University Hospital, for her help in editing the manuscript.

Contributors OV, SD, TL and GA were involved in the conception and the design of this study. OV, SD, SP and TL were involved in the acquisition of clinical data. 
MK, JN, PR and NS performed the ultrasonographic assessments. OV and GA were involved in the statistical analysis. OV, SD, TL and GA were involved in the analysis and interpretation of the data. All authors were involved in the drafting and revision of the manuscript.

Funding The authors have not declared a specific grant for this research from any funding agency in the public, commercial or not-for-profit sectors.

Competing interests None declared.

Patient consent for publication Not required.

Ethics approval The study (E2014-28) was approved by the local institutional review board (named Ethics Committee for Non-Interventional Research) according to law $n^{\circ} 2012-300$.

Provenance and peer review Not commissioned; externally peer reviewed.

Data availability statement All data relevant to the study are included in the article or uploaded as supplementary information.

Open access This is an open access article distributed in accordance with the Creative Commons Attribution Non Commercial (CC BY-NC 4.0) license, which permits others to distribute, remix, adapt, build upon this work non-commercially, and license their derivative works on different terms, provided the original work is properly cited, appropriate credit is given, any changes made indicated, and the use is non-commercial. See: http://creativecommons.org/licenses/by-nc/4.0/.

ORCID iD

Olivier Vittecoq http://orcid.org/0000-0001-9268-745X

\section{REFERENCES}

1 Pinals RS, Masi AT, Larsen RA, et al. Preliminary criteria for clinical remission in rheumatoid arthritis. Arthritis Rheum 1981;24:1308-15.

2 Ometto F, Botsios C, Raffeiner B, et al. Methods used to assess remission and low disease activity in rheumatoid arthritis. Autoimmun Rev 2010;9:161-4.

3 Tanaka Y, Hirata S, Saleem B, et al. Discontinuation of biologics in patients with rheumatoid arthritis. Clin Exp Rheumatol 2013;31:S22-7.

4 Smolen JS, Landewé R, Breedveld FC, et al. EULAR recommendations for the management of rheumatoid arthritis with synthetic and biological disease-modifying antirheumatic drugs: 2013 update. Ann Rheum Dis 2014;73:492-509.

5 Zhang B, Combe B, Rincheval N, et al. Validation of ACR/EULAR definition of remission in rheumatoid arthritis from RA practice: the ESPOIR cohort. Arthritis Res Ther 2012;14.

6 Felson DT, Smolen JS, Wells G, et al. American College of Rheumatology/European League against rheumatism provisional definition of remission in rheumatoid arthritis for clinical trials. Arthritis Rheum 2011;63:573-86.

7 Tweehuysen L, van den Ende $\mathrm{CH}$, Beeren FMM, et al. Little evidence for usefulness of biomarkers for predicting successful dose reduction or discontinuation of a biologic agent in rheumatoid arthritis: a systematic review. Arthritis Rheumatol 2017;69:301-8.

8 D'Agostino M-A, Boers M, Wakefield RJ, et al. Exploring a new ultrasound score as a clinical predictive tool in patients with rheumatoid arthritis starting abatacept: results from the APPRAISE study. RMD Open 2016;2:e000237.

9 Szkudlarek M, Wakefield RJ, Backhaus M, et al. The discriminatory capacity of ultrasound in rheumatoid arthritis: active vs inactive, early vs advanced, and more. Rheumatology 2012;51:vii6-9.

10 Smolen JS, Nash P, Durez P, et al. Maintenance, reduction, or withdrawal of etanercept after treatment with etanercept and methotrexate in patients with moderate rheumatoid arthritis (preserve): a randomised controlled trial. The Lancet 2013;381:918-29.
11 van den Broek M, Klarenbeek NB, Dirven L, et al. Discontinuation of infliximab and potential predictors of persistent low disease activity in patients with early rheumatoid arthritis and disease activity scoresteered therapy: subanalysis of the BeSt study. Ann Rheum Dis 2011;70:1389-94.

12 Brocq O, Millasseau E, Albert C, et al. Effect of discontinuing TNF $\alpha$ antagonist therapy in patients with remission of rheumatoid arthritis. Joint Bone Spine 2009;76:350-5.

13 Emery P, Hammoudeh M, FitzGerald O, et al. Sustained remission with etanercept tapering in early rheumatoid arthritis. N Engl J Med 2014;371:1781-92.

14 Fautrel B, Gandjbakhch F, Foltz V, et al. OP0066 Targeting the lowest efficacious dose for rheumatoid arthritis patients in remission: clinical and structural impact of a step-down strategy trial based on progressive spacing of TNF-Blocker Injections (Strass Trial). Ann Rheum Dis 2013.

15 Tanaka Y, Takeuchi T, Mimori T, et al. Discontinuation of infliximab after attaining low disease activity in patients with rheumatoid arthritis: RRR (remission induction by remicade in RA) study. Ann Rheum Dis 2010;69:1286-91.

16 Saleem B, Keen H, Goeb V, et al. Patients with RA in remission on TNF blockers: when and in whom can TNF blocker therapy be stopped? Ann Rheum Dis 2010;69:1636-42.

17 Balsa A, de Miguel E, Castillo C, et al. Superiority of SDAl over DAS28 in assessment of remission in rheumatoid arthritis patients using power Doppler ultrasonography as a gold standard. Rheumatology 2010;49:683-90.

18 Gärtner M, Alasti F, Supp G, et al. Persistence of subclinical sonographic joint activity in rheumatoid arthritis in sustained clinical remission. Ann Rheum Dis 2015;74:2050-3.

19 Nguyen H, Ruyssen-Witrand A, Gandjbakhch F, et al. Prevalence of ultrasound-detected residual synovitis and risk of relapse and structural progression in rheumatoid arthritis patients in clinical remission: a systematic review and meta-analysis. Rheumatology 2014:53:2110-8.

20 Naredo E, Valor L, De la Torre I, et al. Predictive value of Doppler ultrasound-detected synovitis in relation to failed tapering of biologic therapy in patients with rheumatoid arthritis. Rheumatology 2015;54:1408-14.

21 Iwamoto T, Ikeda K, Hosokawa J, et al. Prediction of relapse after discontinuation of biologic agents by ultrasonographic assessment in patients with rheumatoid arthritis in clinical remission: high predictive values of total gray-scale and power Doppler scores that represent residual synovial. Arthritis Care Res 2014;66:1576-81.

22 Alivernini S, Peluso G, Fedele AL, et al. Tapering and discontinuation of TNF- $\alpha$ blockers without disease relapse using ultrasonography as a tool to identify patients with rheumatoid arthritis in clinical and histological remission. Arthritis Res Ther $2016 ; 18$.

23 Scott IC, Kingsley GH, Scott DL. Can we discontinue synthetic disease-modifying anti-rheumatic drugs in rheumatoid arthritis? Clin Exp Rheumatol 2013;31:S4-8.

24 Deighton C, O'Mahony R, Tosh J, et al. Management of rheumatoid arthritis: summary of NICE guidance. BMJ 2009;338:b702.

25 Schett G, Emery P, Tanaka Y, et al. Tapering biologic and conventional DMARD therapy in rheumatoid arthritis: current evidence and future directions. Ann Rheum Dis 2016;75:1428-37.

26 Rech J, Hueber AJ, Finzel S, et al. Prediction of disease relapses by multibiomarker disease activity and autoantibody status in patients with rheumatoid arthritis on tapering DMARD treatment. Ann Rheum Dis 2016;75:1637-44.

27 Baker KF, Skelton AJ, Lendrem DW, et al. Predicting drug-free remission in rheumatoid arthritis: a prospective interventional cohort study. J Autoimmun 2019;105:102298.

28 Tasaki S, Suzuki K, Kassai Y, et al. Multi-omics monitoring of drug response in rheumatoid arthritis in pursuit of molecular remission. Nat Commun 2018;9:2755. 